Abstract

\title{
Beyond Nutrition: From Conceptualization to Development-A Case Study of a Bread ${ }^{+}$
}

\author{
Isaac Amoah ${ }^{1,2}$, Carolyn Cairncross ${ }^{1}$ and Elaine Rush ${ }^{1,2, *}$ \\ 1 Faculty of Health and Environmental, Auckland University of Technology, Auckland 1010, New Zealand; \\ isaac.amoah@aut.ac.nz (I.A.); carolyn.cairncross@aut.ac.nz (C.C.) \\ 2 Riddet Institute, Centre of Research Excellence, Palmerston North 4442, New Zealand \\ * Correspondence: elaine.rush@aut.ac.nz; Tel.: +64-21-624-077 \\ + Presented at the 2019 Annual Meeting of the Nutrition Society of New Zealand, Napier, New Zealand, \\ 28-29 November 2019.
}

Published: 13 December 2019

As well as obtaining nourishment from bread, consumer acceptability is dependent on the appearance, texture, aroma, taste and swallowablity. A nutrient-dense vegetable-enriched bread (VB) was produced and in a series of 3 experimental studies physical properties, sensory attributes, ease of swallowing, glycaemic response, satiety, lipid profile and plasma $\beta$-Carotene concentration response of this bread compared to commercial white (WB) and wheatmeal breads (WMB).

Objective properties including texture and bread crumb colour were measured. The liking and swallowing attributes were subjectively evaluated by older adults $(n=50,50+y)$ using Likaert scales. Subsequently young participants $(\mathrm{n}=10,23 \pm 7 \mathrm{y})$ were randomised to consume $75 \mathrm{~g}$ of one of VB, WB and WMB after an overnight fast on three separate days. Blood samples before and 15, 30, 45, 60, 90, $120 \mathrm{~min}$ after consumption were analysed for glucose and insulin concentration. Satiety questionnaires were completed at each sampling point. The incremental-area-under-the-curves (iAUC) for glucose and insulin were calculated. Lastly, 10 participants $(n=10,30 \pm 12 y)$ consumed $200 \mathrm{~g}$ of VB every day for two weeks. Plasma $\beta$-carotene and lipid profiles were measured pre and post.

Participants preferred the vegetable-enriched bread and reported that it was easier to swallow than WB and WMB. The glucose iAUC were not different among breads but the mean insulin iAUC for the VB was significantly less than the WB and WMB; WB minus VB difference $12415 \mathrm{pmol} \cdot \mathrm{min} / \mathrm{L}$ $(95 \% \mathrm{CI} 1918,22912 \mathrm{pmol} \cdot \mathrm{min} / \mathrm{L}, p=0.025)$ and WMB minus VB $13800 \mathrm{pmol} \cdot \mathrm{min} / \mathrm{L}(95 \% \mathrm{CI} 1623,25976$ $\mathrm{pmol} \cdot \mathrm{min} / \mathrm{L}, p=0.031$ ). The VB satisfied hunger more than the WB and WMB. The consumption of VB for two weeks did not statistically change plasma $\beta$-Carotene and plasma lipid profile.

The vegetable-enriched bread attenuated insulin release, provided greater satiety and was favored over the commercial breads. A longer trial is warranted.

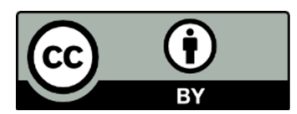

(C) 2019 by the authors. Licensee MDPI, Basel, Switzerland. This article is an open access article distributed under the terms and conditions of the Creative Commons Attribution (CC BY) license (http://creativecommons.org/licenses/by/4.0/). 